\title{
COMBINING WLI AND SEM TECHNIQUES TO OBTAIN A 4D SURFACE IMAGE OF A PpHDMSO/AICeO ${ }_{3}$ NANOCOMPOSITE
}

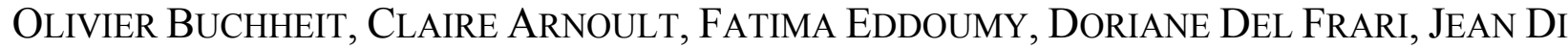 \\ MARTINO AND DAVID RUCH
}

Laboratoire des Technologies Industrielles, CRP Henri Tudor, Rue de Luxembourg 66, BP 144, L-4002 Eschsur-Alzette, Luxembourg

e-mail: olivier.buchheit@tudor.lu

(Accepted October15, 2009)

\begin{abstract}
Compositional images from a SEM (scanning electron microscope) are sometimes complemented by quantitative topographical data from devices such as an AFM (atomic force microscope) or WLI (white light interferometer). Indeed, even if a SEM could provide both kinds of information (composition and topography), the topographical data are incomplete because the SEM does not allow measuring the vertical dimension (i.e., perpendicular to the measurement plane). Thus these two kinds of information are usually measured using two different techniques, and at different locations on the sample. Mean values of surface composition are then linked to mean values of topography, and as a consequence this approach does not allow precisely linking a local topographical peak to its corresponding composition. The present work deals with a SEM/WLI combination methodology, based on the characterization, at the same location, of a nanocomposite (nanoparticles of $\mathrm{AlCeO}_{3}$ dispersed in a plasma-polymerized hexamethyldisiloxane ppHMDSO matrix and deposited by atmospheric plasma on a glass substrate) developed for improving anticorrosion properties. SEM images allow the supposition that the protuberances (peaks) observed on the surface of a specimen are linked to the nanoparticles dispersed in the polymer coating, but this link is not fully convincing. Thanks to a precise localization method, SEM compositional data and WLI topographical data are here measured at the same location. The recombination of both signals to form a 4D image (3D geometry and 1D composition) allows linking protuberances to nanoparticles aggregates without ambiguity. This composite image appears to be an interesting new tool (at the scale of observation, i.e., hundreds of micrometers) for the study of nanostructured coatings.
\end{abstract}

Keywords: composition mode imaging, cross-analysis, nanoparticles dispersion, scanning electron microscopy, topography, white light interferometry.

\section{INTRODUCTION}

Nowadays, multi-technique approaches are widely used to characterize a material, especially in the field of surface characterization. For instance, the knowledge of both analytical and topographical information can be of great interest when dealing with contacting surfaces (tribology), fractography, surface functionalization, optimization of a fabrication process, etc. Several techniques are available to access such information: see for example Leopold et al. (2003) reporting topographical analysis of sheet metals with the projected fringes method (PFM), white-light interferometry (WLI), laser scanning methods (LSM), structuredlighting reflection technique (SLRT), scanning electron microscopy (SEM) and atomic force microscopy (AFM). For chemical information, several tools are also available: $\mathrm{x}$-ray spectrometry (x-ray energy dispersive spectroscopy EDS or electron microprobe EMP), auger electron spectroscopy (AES), x-ray fluorescence (XRF), secondary ion mass spectrometry (SIMS), etc. But none of these techniques gives simultaneous access to both numerical topographical data (over the 3 dimensions of space) and chemical data at the location of interest.

The SEM technique is very versatile, providing information on surface composition, morphology, porosity, layer thickness, etc. In addition, this technique offers great magnification, important depth of field and high resolution; however, the SEM topographical mode is mainly qualitative. In that mode, image contrast is based on detection of the secondary electrons (SE) issued from the specimen. The image contrast results from a combination of many physical processes and depends on the instrumental set-up (mainly on local changes of incident beam angle and 
correlated changes of detection angle (Cazaux, 2008), with the beam energy determining the relative weight of each contribution). This can be understood as a process in which the relative topographical contrast between two areas depends on the local surface slope, not on local surface height; two areas with equal slope will induce equal contrast on SEM measurements, without a link to their relative heights. Thus, it is impossible to quantify topographical functions along the axis perpendicular to the measurement plane. It is technically possible to build a numerical $3 \mathrm{D}$ surface (i.e., stereometry) with SEM thanks to specific software, but topography and chemical contrast still cannot simultaneously be accessed. Successive measurements on the same area are then needed, implying a longer exposure time to the SEM beam, and as a result possible surface damage. Indeed, significant damage can occur on organic and inorganic samples (Eggerton et al., 2004) when exposed to an electron beam; one major effect is the modification of the sample's surface morphology. This can be problematic when working on polymers that are very sensitive to energetic irradiation (PMMA, PP, etc.).

Thus, chemical and qualitative topographical data from SEM are sometimes combined with quantitative information obtained from AFM measurements. Elimelech et al. (1997) coupled contact mode AFM and SEM measurements to demonstrate the influence of the roughness of membranes on fouling by a colloidal suspension of silica. Gsell et al. (2007) studied the diamond nucleation layer on Iridium (001): AFM and SEM were combined to form images of a small identical area on the sample. In the biological field, Ubbink and Schär-Zammaretti (2005) worked on bacterial interactions; integration of electron microscopy and AFM data is discussed for various bacterial strains. However, the area scanned by AFM is small (around one hundred square micrometers), with a depth of field of some micrometers. Consequently, roughness values obtained by AFM can be misleading, being evaluated over an area too small with regard to the studied problematic. As an example, Koyuncu et al. (2006) showed that roughness values obtained by WLI were higher than those obtained by AFM, highlighting the importance of the observation scale, i.e., of the measurement device, when dealing with surface topography. Furthermore, AFM measurements are time consuming.

WLI is a recent technique used to assess surface topography. This non-contact method quickly measures the 3D geometry of surfaces. Based on the reflection from the measured surface of a white light beam into an interferometric objective, this technique provides quantitative information for much larger areas (up to some square millimetres) with a vertical resolution around ten nanometers. Thus, WLI is another technique that can be used for height measurement in the vertical direction, along with complete morphological and analytical data from SEM (List et al., 2005; Liu, 2005). However, these combinatorial experiments are usually done at different locations on the sample, hindering the correlation between local composition and corresponding quantitative topography.

The present work deals with a combined SEM/ WLI methodology to correlate topographical and compositional information, using plasma polymer/ nano $\mathrm{AlCeO}_{3}$ composites as an example. According to new legislation (European directives, 2000, 2002), wet chemical processes for metal passivation based on hexavalent chromium $\mathrm{Cr}^{\mathrm{VI}}$ are banned due to their toxicity. The challenge, then, is to obtain by a dry process a material exhibiting the same level of resistance to corrosion. Mixing of plasma-polymerized hexamethyldisiloxane (ppHMDSO), known to be a promising way to achieve physical barrier coatings, and cerium nanoparticles, known to have interesting electroactive properties, is a promising way to reproduce the anticorrosion properties of $\mathrm{Cr}^{\mathrm{VI}}$-based coatings. In this context, innovative layers of organosilicon matrix and $\mathrm{AlCeO}_{3}$ particles have been developed. Here, the mixture of both components is atomized and introduced as precursors into an atmospheric pressure dielectric barrier discharge (DBD) where plasma polymerization of the organosilicon precursor occurs (Bardon et al., 2007); this fabrication process is totally free of solvents.

Previous studies were carried out on samples with different nanoparticle concentrations, and their morphologies were respectively studied by SEM and WLI (Del Frari et al., 2010). SEM investigations in compositional contrast showed that many nanoparticles formed aggregates, more or less spherical, that can reach some micrometers in diameter. In addition, an excess thickness within the matrix (protuberances) was sometimes visible, but the direct link between the nanoparticles and these peaks could not strictly be established, as topography and composition were not measured at the same location. Thus, we focus here on the nature of these protuberances. The superposition of composition information (SEM) and topographical data (WLI) in a 4D image (three dimensions of space and 1 dimension of composition) will allow confirmation of the correlation between nanoparticle aggregates and protuberances. It will then be possible to better study the relationship between surface 
roughness and the concentration of nanoparticles in the precursor (liquid), and will provide information regarding the distribution of nanoparticles inside the matrix.

\section{MATERIALS AND METHODS}

\section{SUBSTRATES AND SOLUTIONS}

Substrates are glass slides degreased with ethanol. $\mathrm{AlCeO}_{3}$ nanoparticles (Aldrich, 99\%) are made of some aggregates with diameters varying between a few $\mathrm{nm}(<25 \mathrm{~nm})$ and $300 \mathrm{~nm}$. They are added to a hexamethyldisiloxane (HMDSO $>98 \%$ grade) solution, purchased from Aldrich. Solutions are homogenized by ultrasonic stirring at room temperature for $30 \mathrm{~min}$. Five samples were studied according to nanoparticle concentration in the atmospheric plasma precursor (percent by weight): sample 0 for $0 \%$ of $\mathrm{AlCeO}_{3}$, sample 1 for $1 \%$ of $\mathrm{AlCeO}_{3}, \ldots$ sample 4 for $4 \%$ of $\mathrm{AlCeO}_{3}$.

\section{PLASMA DEPOSITION PROCESS}

Coating deposition was performed in a semidynamic dielectric barrier discharge (DBD) reactor. The liquid precursor solution was atomized at a constant atomization pressure of $2 \times 10^{5} \mathrm{~Pa}$ and injected into a carrier gas flow before entering the plasma zone. A gas mixture containing the precursor aerosol was injected into the plasma through a slit between the two top electrodes. The coating was carried out at atmospheric pressure and ambient temperature. The resulting thickness of the coating ranged from $150 \mathrm{~nm}$ to $400 \mathrm{~nm}$.

\section{WHITE LIGHT INTERFEROMETER}

Three-dimensional topological data were determined using a white light interferometer (Wyko NT 3300, Veeco). Experimental parameters were $0.55 \mu \mathrm{m}$ of lateral resolution, $10 \mathrm{~nm}$ of vertical resolution, vertical shifting interferometry (VSI) mode, VSI filter, and measurement size in the X-Y plane of approximately 60 by $50 \mu \mathrm{m}$. The stitching mode was used to extend the measurement area to 150 by $150 \mu \mathrm{m}$, with a $20 \%$ overlap.

\section{SCANNING ELECTRON MICROSCOPY}

SEM analyses were carried out on a QUANTA 200 FEG from FEI equipped with an X GENESIS XM 4i EDS spectrometer supplied by EDAX. This microscope is a variable pressure SEM (VP-SEM) that enables direct observation of insulating samples without additional coating (Robinson, 1975; Danilatos, 1993). Analyses were performed by varying the specimen chamber pressure from a high vacuum $\left(10^{-3}\right.$ $\mathrm{Pa}$ ) to $200 \mathrm{~Pa}$, depending on the charging effect. The gas introduced was water vapour.

As topographical information is obtained from WLI, SEM experimental conditions were optimized to get the best chemical contrast and clearly locate the $\mathrm{AlCeO}_{3}$ fillers inside the polymer matrix. Imaging was performed using the backscattered electron (BSE) signal, whose intensity depends on the local composition: areas with higher average atomic number $\left(\mathrm{AlCeO}_{3}\right.$ fillers) appear in lighter grey, whereas areas with lower average atomic number (polymer matrix) appear in darker grey. BSE images were obtained with a $10 \mathrm{kV}$ electron probe, which enables good BSE contrast and limits polymer degradation. Under these experimental conditions, the whole thickness of the coating was probed. The resulting image corresponds to a $2 \mathrm{D}$ projection of all the fillers present in the coating. The dimensions of the projection are then directly linked to the real transverse dimension of the fillers, without being affected by their position in the thickness of the coating. Samples were cleaned by air projection before measurement to remove surface contamination.

\section{CO-REGISTRATION OF SEM AND WLI MEASUREMENTS}

A basic and quick method of sample positioning was defined to perform both SEM and WLI analyses on the same area of the sample.

Positioning errors must be minimized to enable the largest area of coverage between both methods and minimize the correction done in software. Three spatial parameters are necessary for the sample. First, a physical point used as the origin. This point eliminates translations between SEM and WLI measurements. Then, at least one line is necessary to eliminate rotation errors (two is optimal). These two parameters ensure identical positioning of measurement areas. Finally, two directions, along both the reference line and a perpendicular axis, are defined for orientation of the mapping.

Thus, our markers are two perpendicular lines on the surface of the sample. The origin is the intersection of these lines, and the directions are chosen to go from left to right and from bottom to top. As the positioning on sample holders is done manually, it is necessary to make measurements as close to the markers as possible to reduce the effect of unavoidable small misalignments. Ideally, the markers are included 
in the measured area and are cropped during image analysis.

Many trials were performed to find the best way to create these marker lines. Topographical marks such as scratches did not give proper results: damage quickly appears on track borders, making them impossible to use as references. The best solution is to use two aluminium tapes stuck down perpendicularly on the samples. Easy to set-up, this method has the complementary advantage of helping evacuate the residual electronic charges located in the sample during SEM analysis.

As the measured surface can be degraded by the SEM electron beam, measurement systematically begins with WLI acquisition. The basic WLI images correspond to a field of view of $60 \times 50 \mu \mathrm{m}$. Successive basic images are then acquired and pasted (stitched) to cover the desired measurement field. The stitching operation is done with Vision32 software (the software driving the WLI) with a $20 \%$ overlap on two neighbouring images. As shown in Fig. 1, the first WLI image is positioned so that the aluminium tape landmark is located in the lower left-hand corner of the measurement area. Mapping is then realized as a matrix of $3 \times 4$ basic images, corresponding to a general field of view of ca. $175 \times 175 \mu \mathrm{m}$.

SEM mapping is "included" in the WLI mapping (see Fig. 1). A first general location is set with a single SEM image of $500 \times 500 \mu \mathrm{m}(1024 \times 884$ pixels) to horizontally and vertically position the aluminium landmarks. After this "rough" positioning, a focus is made on the landmark's origin point (image of ca. $60 \times 50 \mu \mathrm{m}, 1024 \times 884$ pixels). This focus allows optimizing the positioning alignment. Then, a SEM starting image is obtained by zooming in on the centre of the previous SEM view (i.e., image of $30 \mathrm{x}$ $25 \mu \mathrm{m}, 1024 \times 884$ pixels). Finally, a series of micrographs is taken along a $5 \times 5$ matrix field of view, by translation along both axes with an overlapping area of $15 \%$. With this last setup, the best lateral experimental resolution achieved (i.e., the finest nanoparticle agglomerate that is distinguished) is around $100 \mathrm{~nm}$, which is better than the resolution obtained with WLI. The whole 4D view of $100 \times 100$ $\mu \mathrm{m}$ is then built by visually stitching WLI and SEM images thanks to common topographical/surface functions present on overlapping areas.

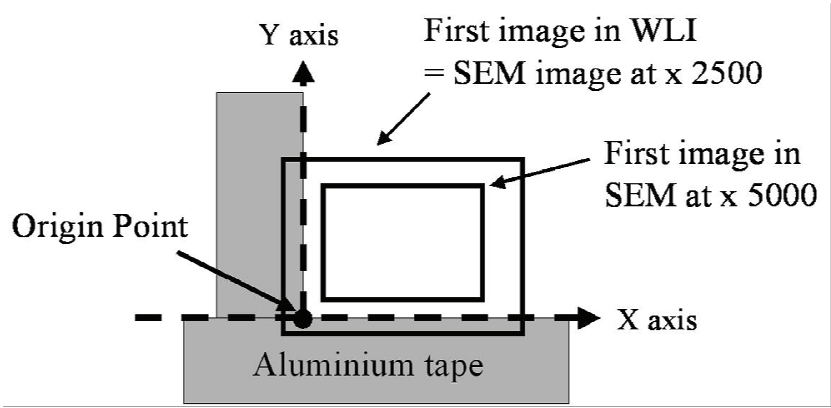

Fig. 1. Measurement methodology: drawing of a common datum, used for both techniques, on the sample surface.

\section{DATA PROCESSING}

Topographical data (or surfometry) from white light interferometer are analyzed with MountainsMap 5.0.3 software (Digital Surf, 2008). The treatment procedure consists in filling in non-measured points of the surfometry, removing form defects with a $3^{\text {rd }}$ order polynomial, and thresholding the AbbottFirestone curve (the integral of the amplitude distribution function, see Abbott and Firestone, 1933) to remove extreme, aberrant data points (these points, related for example to measurement noise, are seen as Dirac functions on the topography).

Composition images from the SEM are processed using GIMP 2.4.2 software (Gimp, 2008). Nanoparticle sites are first highlighted using a contour analysis, Sobel algorithm of quantity 1, spread-out. After this discrimination, nanoparticle agglomerates become well defined, but small-intensity pollution of the contour analysis remains; a threshold is then applied to remove this pollution. Binarization of the image follows: only one colour for the polymer, and another one for the nanoparticles. The processed SEM image is then pasted as a texture on the topographical signals in MountainsMap, using the texture option.

\section{RESULTS}

Fig. 2 presents the 4D images obtained on three samples. Each image is a combination of the topographical signal, giving the sample's surface geometry, and of the composition signal, defined by the colour (either white or red) on the surface. The white colour is linked to the plasma-polymerized 
HMDSO matrix and the red colour is relative to the $\mathrm{AlCeO}_{3}$ nanoparticles, except for the red lines: these are residues from the outline step during SEM image processing, linked to the charging effect during SEM measurement, and therefore should not be considered. The black colour is the shadow of the peaks, allowing better clarity.

The reference sample (Fig. 2a) exhibits local topographical heterogeneities (peaks). These are linked to surface contamination: the reference sample is supposed to be more electrostatic than the others and thus is less sensitive to the air-cleaning procedure. The red signal on these peaks is linked to their composition (higher average atomic number than the polymeric matrix), but is not relative to $\mathrm{AlCeO}_{3}$. For $1 \%$ of nanoparticles, we see an important increase of both the number of peaks and $\mathrm{AlCeO}_{3}$ signals, the latter being very localized (small dots and discs). This phenomenon increases with increasing nanoparticle concentration (Fig. 2c). The size of the filler signals (up to some micrometers in diameter), greater than the expected size of the nanoparticles, confirms that nanoparticles are aggregated in the polymeric matrix, forming small islands of various size (see the zoomed view on Fig. 2d), instead of staying isolated and equally dispersed in the matrix.
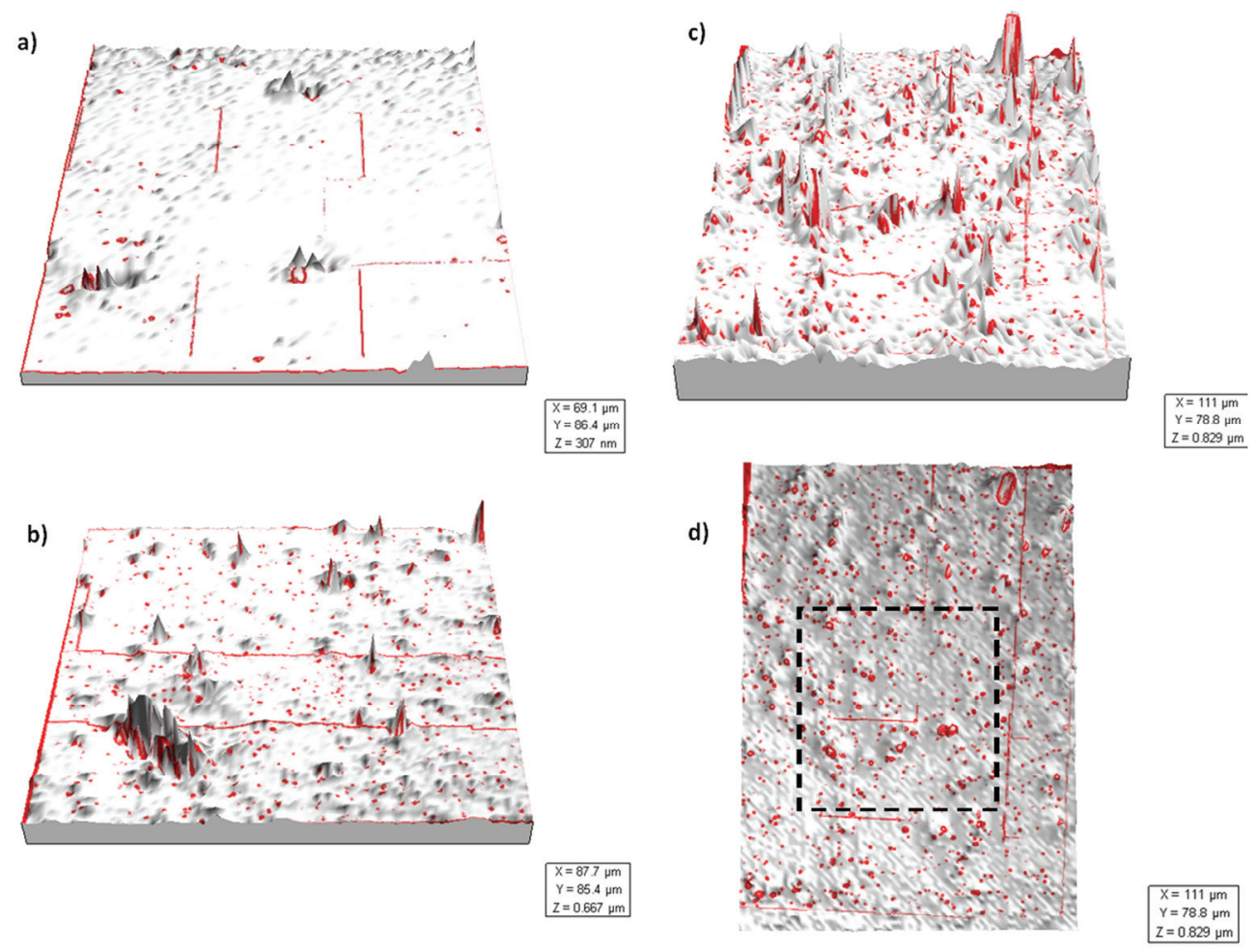

$X=87.7 \mu \mathrm{m}$
$\gamma=85.4 \mu \mathrm{m}$
$Z=0.667 \mu \mathrm{m}$

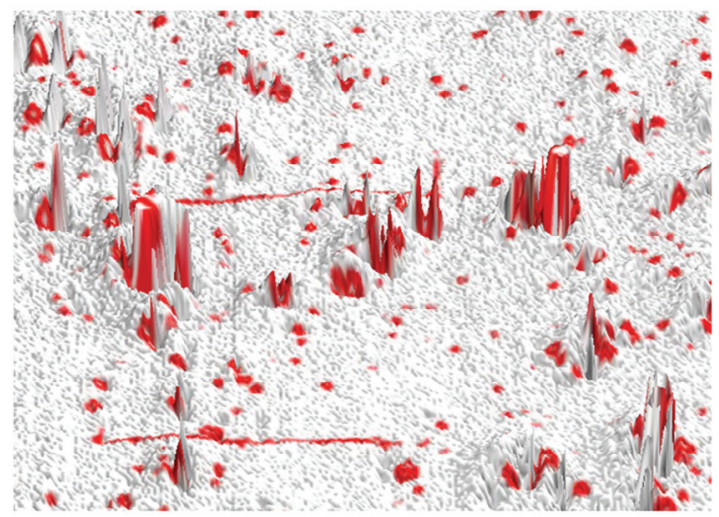

Fig. 2. $4 \mathrm{D}$ representations of ppHDMSO(matrix, white)/AlCeO $\mathrm{O}_{3}$ (nanoparticles, red) samples. a) Tilted view of sample $0 \% \mathrm{AlCeO}_{3}$ nanoparticles, b) $1 \%$, c) 3\%, d) Top view of sample 3\%. The black box in dotted line indicates the zoomed area given in e). e) Tilted view of sample 3\% zoomed. Area of $48 \mu m$ (X-axis) $x 46 \mu m(Y$ axis). 


\section{DISCUSSION}

It is now obvious from the 4D images that peaks are directly linked to the presence of nanoparticles: each peak is present over or very close to a nanoparticle agglomerate signal. Bigger peaks are linked to wider aggregate signals, as shown in Figs. 2 d,e. This observation is confirmed by SEM crosssection imagery (Fig. 3): the large peak observed on the surface is directly linked to the underlying nanoparticle aggregate.

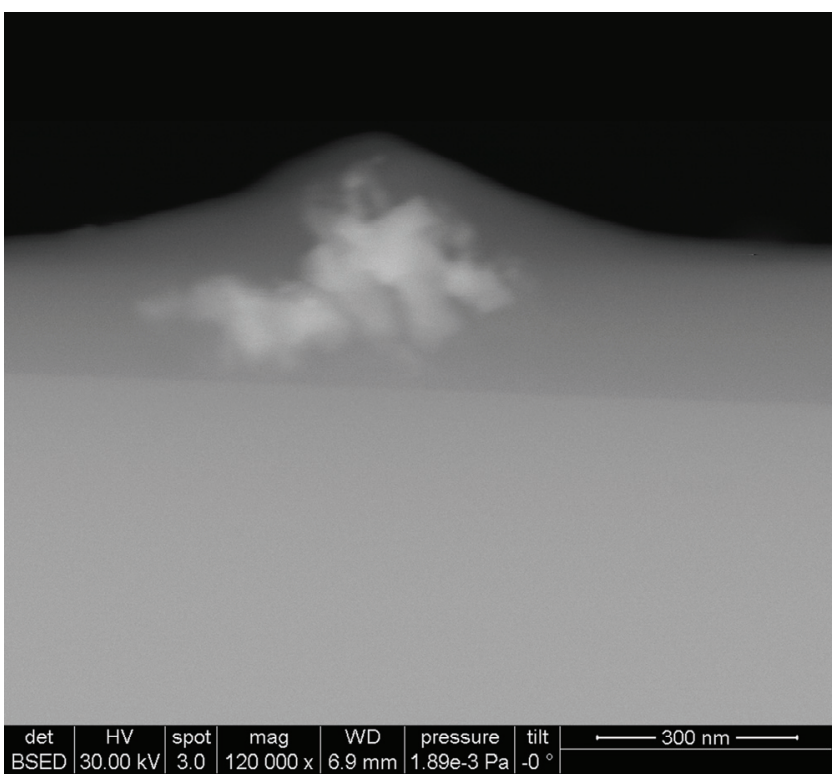

Fig. 3. Backscattered electron picture, cross-section, sample 3. The nanoparticles appear in clear white, surrounded by the polymeric matrix deposited on silicon substrate.

The 4D reconstruction gives complementary information: Figs. 2d,e clearly show that the appearance of topographical peaks is linked to a "critical" size of nanoparticle agglomerates. Indeed, there are numerous small composition dots surrounding bigger ones; these small dots are not associated with repeatable topographical variations (dots are sometimes on small hills, sometimes in small valleys). But all the larger nanoparticle agglomerates are linked to topographical peaks. The link between the size of the peak, the size of the underlying nanoparticle agglomerate and the thickness of the coating will be further investigated. Nevertheless, to illustrate the interest of the 4D approach, we present here preliminary results of peak shape. Similar results were presented in a preliminary work (Del Frari et al., 2010), but the numerical values were different due to differences in both resolution of topographical measurements and data processing. Despite this difference in absolute values, the relative evolution of the shape of peaks with nanoparticle concentrations is the same. Here, topographical measurements were thresholded based on the value of the $\mathrm{Srl}$ parameter derived from the Abbott-Firestone curve; this parameter, called the upper material ratio, is directly linked to the upper part of the profile (the peaks), allowing isolating them from the entire profile. Once isolated, the peaks were filtered using a $9 \times 9$ median filter to remove very small peaks and unrepresentative noise; then the mean volume, height, surface and height-over-surface ratio of the remaining peaks (i.e., peaks related to agglomerates of nanoparticles) were calculated. The results are given in Table 1. We see that mean volume, surface and height increase with increasing nanoparticle concentration, excepted for the $2 \%$ concentration. This exception has still to be explained; current advances lead us to consider this as an outlier due to the coating process, but it could also be linked to a peculiar nanoparticle concentration where agglomerate behaviour is somewhat different than for other concentrations. Nevertheless, these increases in peak geometry lead us to the supposition that agglomerates become bigger (over the 3 dimensions) when nanoparticle concentration increases.

Table 1. Characterization of peak shape as a function of nanoparticles concentration.

\begin{tabular}{l|c|c|c|c|c}
\hline $\begin{array}{l}\text { Nanoparticles } \\
\text { concentration }\end{array}$ & $\%$ & 1 & 2 & 3 & 4 \\
\hline Mean volume & $\mu \mathrm{m}^{3}$ & 0.207 & 0.163 & 0.311 & 0.495 \\
\hline Mean surface & $\mu \mathrm{m}^{2}$ & 6 & 6 & 7 & 11 \\
\hline Mean height & $\mathrm{nm}$ & 49 & 41 & 87 & 105 \\
\hline $\begin{array}{l}\text { Mean height over } \\
\text { surface ratio }\end{array}$ & $\mathrm{nm} / \mu^{2}$ & 8 & 7 & 12 & 10 \\
\hline
\end{tabular}

The third aspect resulting from this 4D image is that the evolution of topographical parameters is directly linked to nanoparticle concentration: Fig. 4 plots the evolution of two topographical parameters, $\mathrm{Ra}$ and $\mathrm{Sdr}$, as a function of nanoparticle concentration. $\mathrm{Ra}$, the most common topographical parameter, is the arithmetic mean height deviation of 2D roughness profiles. These profiles are obtained by converting the 3D topographical surface into two orthogonal series of profiles (along the $\mathrm{X}$ - and the $\mathrm{Y}$-axis), and applying a Gaussian filter with an $8 \mu \mathrm{m}$ cut-off to each profile of the series. $\mathrm{Ra}$ is then calculated from the $z=f(x)$ signals, regarding the mean line of the profiles (obtained for each profile by subtracting the least mean square line), following the definition:

$$
R a=\frac{1}{L} \int_{L}|z(x)| d x,
$$


$\mathrm{Ra}$ is calculated here along both the $\mathrm{X}$ - and the $\mathrm{Y}$ axis for better appreciation of the average roughness of the surface. Nevertheless, Ra alone is often of little interest to describe the topography of a surface. Here, this bi-dimensional evaluation is completed by the $\mathrm{Sdr}$ parameter, calculated in 3D. Sdr is the developed interfacial area ratio, the ratio of the curvilinear area to the support (i.e., projected) area. This parameter indicates the complexity of the surface, i.e., how far the surface deviates from a perfect plane. A completely flat surface has an Sdr equal to $0 \%$. The more complex the surface, the higher is the $\mathrm{Sdr}$ parameter. Fig. 4 shows that both Ra (over X-and Yaxis) and $\mathrm{Sdr}$ increase with increasing nanoparticle concentration (except for $2 \%$, as previously mentioned). This means that when the concentration of nanoparticles in the precursor increases, the topography of the coated sample becomes more disrupted, rough, with more numerous peaks, of greater amplitude and cross-sectional area.

These three pieces of information - the nature of the peaks, critical size of the agglomerate for the appearance of peaks and evolution of peak geometry/ surface topography with nanoparticle concentration, brought by the 4D image - are of great interest for better understanding and optimization of the deposition process of nanoparticles via atmospheric plasma.

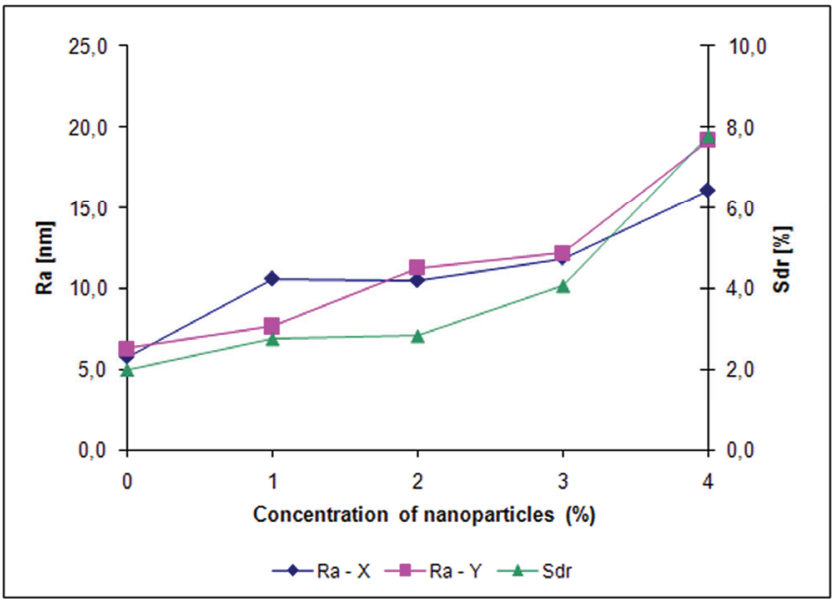

Fig. 4. Graphical representation of $R a$ and $S d r$ parameters as a function of nanoparticles concentration.

\section{CONCLUSION}

In this study, two complementary tools, the white light interferometer (WLI) and the scanning electron microscope (SEM), were combined to study the topographical impact of nanoparticles dispersed in a polymeric matrix some hundred nanometers thick.
Thanks to a basic localization method, it was possible to reconstruct a new 4D image, combining 3D topographical information from the WLI and an "analytical skin" derived from SEM measurements. This recombination allowed proving unambiguously that topographical peaks are due to the presence of underlying agglomerates of nanoparticles. Then, peak geometry as a function of nanoparticle concentration was accessed. Furthermore, the existence of a critical size of nanoparticle agglomerate for topographical modification was identified, but this needs to be studied further. Other foreseen developments concern the optimisation of the couple (roughness parameters/ nanoparticle concentration) regarding end-use product specifications. Another subject of interest is to study the impact of plasma parameters on the aggregation capacity of nanoparticles, with corresponding topographical impact.

\section{ACKNOWLEDGEMENTS}

The authors would like to thank Mr. Fiorucci and Mr. Kloos (Arcelor Mittal Dudelange) for providing galvanized steel samples, and Digital Surf for MountainsMap ${ }^{\circledR}$ software.

\section{REFERENCES}

Abbott EJ, Firestone FA (1933). Specifying surface quality. Mech Eng 55:569-72.

Bardon J, Bour J, Aubriet H, Ruch D, Verheyde B, Dams $\mathrm{R}$, et al. (2007). Deposition of organosilicon-based anticorrosion layers on galvanized steel by atmospheric pressure dielectric barrier discharge plasma. Plasma Process Polym 4:445-9.

Cazaux J (2008). On some contrast reversals in SEM: application to metal/insulator systems. Ultramicroscopy 108:1645-52.

Danilatos GD (1993). Bibliography of environmental scanning electron microscopy. Microsc Res Tech 25: 529-34.

Del Frari D, Bour J, Bardon J, Buchheit O, Arnoult C, Ruch D (2010). Hybrid layers deposited by an atmospheric pressure plasma process for corrosion protection of galvanized steel. J Nanosci Nanotech 10:2611-9.

Digital Surf (2008). Available at URL: http://www. digitalsurf.fr/en/index.html. Accessed on $4^{\text {th }}$ november 2009.

Egerton RF, Li P, Malac M (2004). Radiation damage in the TEM and SEM. Micron 35:399-490.

Elimelech M, Xiaohua Z, Childress AE, Seungkwan H (1997). Role of membrane surface morphology in colloïdal fouling of cellulose acetate and composite aromatic polyamide reverse osmosis membranes. J Membr Sci 127:101-9. 
European directive 2000/53/EC (2000). http://eur-lex. europa. eu/LexUriServ/LexUriServ.do?uri=OJ:L:2000:269:0034: 0042:FR:PDF

European directive 2002/95/CE (2002). URL: http://eurlex.europa.eu/LexUriServ/LexUriServ.do?uri=OJ:L:2003 :037:0019:0023:FR:PDF

Gimp (2008). Available at URL: http://www.gimp.org. Accessed on $4^{\text {th }}$ november 2009.

Gsell S, Schreck M, Benstetter G, Lodermeier E, Strizter B (2007). Combined AFM-SEM study of the diamond nucleation layer on $\operatorname{Ir}(001)$. Diamond \& Related Materials 16:665-70.

Koyuncu I, Brant J, Luttge A, Wiesner MR (2006). A comparison of vertical scanning interferometry (VSI) and atomic force microscopy (AFM) for characterizing membrane surface topography. J Membr Sci 278:410-7.
Leopold J, Günther H, Leopold R (2003). New developments in fast 3D-surface quality control. Measurement 33: 179-87.

List G, Nouari M, Géhin D, Gomez S, Manaud JP, Le Petitcorps Y, et al. (2005). Wear behaviour of cemented carbide tools in dry machining of aluminium alloy. Wear 259:1177-89.

Liu KW (2005). Combinatorial experiment in Ni-Ti thin films by laser interference structuring. Appl Surf Sci 247:550-5.

Robinson VNE (1975). The elimination of charging artifacts in the scanning electron microscope. J Phys 8:638-40.

Ubbink J, Schär-Zammaretti P (2005). Probing bacterial interactions: integrated approaches combining atomic force microscopy, electron microscopy and biophysical techniques. Micron 36:293-320. 